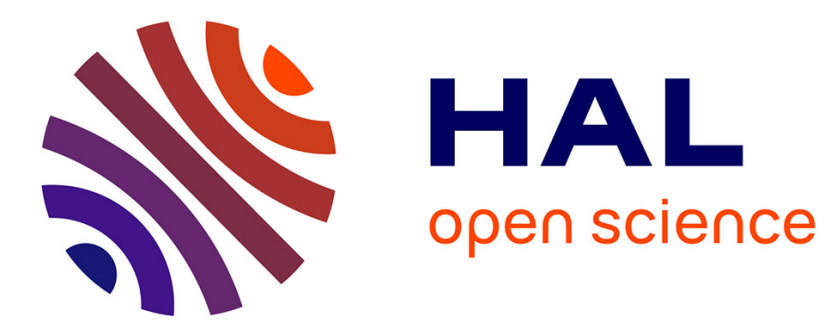

\title{
Il genere e il tempo delle parole: dire la guerra nei testi machiavelliani
}

\author{
Jean-Louis Fournel
}

\section{To cite this version:}

Jean-Louis Fournel. Il genere e il tempo delle parole: dire la guerra nei testi machiavelliani. Del Lucchese, Filippo; Frosini, Fabio; Morfino, Vittorio. The radical Machiavelli : politics, philosophy and language, Brill, pp.23-38, 2015. halshs-01223217

\section{HAL Id: halshs-01223217 https://shs.hal.science/halshs-01223217}

Submitted on 29 Dec 2015

HAL is a multi-disciplinary open access archive for the deposit and dissemination of scientific research documents, whether they are published or not. The documents may come from teaching and research institutions in France or abroad, or from public or private research centers.
L'archive ouverte pluridisciplinaire HAL, est destinée au dépôt et à la diffusion de documents scientifiques de niveau recherche, publiés ou non, émanant des établissements d'enseignement et de recherche français ou étrangers, des laboratoires publics ou privés. 


\section{Il genere e il tempo delle parole: dire la guerra nei testi machiavelliani}

Jean-Louis Fournel

Université Paris 8 et Institut universitaire de France, UMR 5206 Triangle

\section{Preambolo: la lingua dello stato di guerra ${ }^{1}$}

La scrittura della guerra non è una scrittura come le altre nella misura in cui supera le logiche strettamente cognitive o narrative, giacché coinvolge spesso un'intera visione del mondo, con un tentativo di razionalizzare uno spazio/tempo già scombussolato dal conflitto. Soprattutto, essa è animata per lo più da una necessità che porta a confondere analisi e azione: parlare di guerra significa toccare una forma di radicalità perché si tratta sempre di parlare di morte, morte di uomini e morte di Stati. La cosa si complica poi per il fatto che, non senza contraddizione, il racconto della guerra è sempre lo stesso e sempre lacunoso. È sempre uguale perché il racconto di guerra è la base di tutti i racconti epici e storici e, in quanto tali, tornano frequentemente gli stessi codici, gli stessi moduli, gli stessi riferimenti: in questo modo, ogni guerra narrata è spesso una ripetizione di tutte le guerre note nel passato, le quali servono da modelli e da fonti per capire e dire. È sempre lacunoso, perché risulta sempre molto complicato mettere insieme tutte le informazioni necessarie ad un simile racconto e molte cose si possono solamente ipotizzare: si pensi per esempio a quanto è detto sul numero dei morti nelle battaglie a proposito dello scontro di Ravenna nella Storia d'Italia di Francesco Guicciardini (X, 13). Ma quei racconti sono anche unici, perché ogni guerra, per certi versi, non assomiglia a nessun'altra: la guerra sorprende, distrugge, cancella, trasforma e ha sempre una sua componente inaudita e difficile da descrivere.

\footnotetext{
${ }^{1}$ Il presente contributo si inserisce in uno studio sviluppatosi da tempo e per lo più a quattro mani con JeanClaude Zancarini nell'ambito dei nostri lavori di traduzione e di commento di alcune delle opere maggiori della Firenze delle guerre d'Italia. Per questa Firenze ho coniato l'espressione 'repubblica di guerra' (cfr. Fournel 2009). Quanto al nostro lavoro di traduzione, si vedano Guicciardini 1988, e 1996, e 1997, nonché Machiavelli 2000 (nuova edizione rivista: Machiavelli 2014). Questo lavoro è anche illustrato ultimamente dalle voci 'militari' ('Armi', 'Artiglieria', 'Cavalleria', 'Fanteria', 'Fortezze', 'Pace/guerra', 'Nemico', 'Odio/amore', 'Tirannide') redatte da noi due per Sasso 2014. Si veda anche Fontaine e Fournel 2014.
} 
La scrittura della guerra si presta quindi ad un processo di modellizzazione sistematico ma variegato, a seconda degli strumenti e delle forme retoriche predilette, siano esse monumentali o testuali, poetiche o storiografiche, funzionali oppure ritualizzate e celebrative. Di fronte al pericolo della semplice ripetizione, una delle soluzioni è quindi di ricorrere all'analogia, la quale consente di dire il nuovo senza staccarlo del tutto dal passato. Moduli interpretativi legati ad una doxa sovrabbondante si mescolano quindi con la richiesta di una precisione del lessico e della descrizione: la guerra ha a che fare con un sapere costituito per capitani e principi. E quando Machiavelli insiste sul fatto che essa non è un'arte, l'enunciato si iscrive nell'ambito della sua - strutturante - critica dei mercenari: i soldati non devono avere la guerra per arte in nome di motivi quindi spesso più ideologici e politici - l'elogio del cittadino-soldato e delle armi proprie - che gnoseologici. ${ }^{2}$ E ciò non toglie che, per il Fiorentino, chi dirige l'esercito, ossia il principe, 'debbe [...] non avere altro obietto né altro pensiero, né prendere cosa alcuna per sua arte, fuora della guerra et ordini e disciplina di essa; perché quella è sola arte che si espetta a chi comanda', come richiede il capitolo 24 del Principe.

Il racconto di guerra si complica poi ulteriormente per il fatto che accade che spunti in esso l'ammissione del carattere parzialmente indicibile del conflitto armato: quando il 28 maggio 1527, tre settimane dopo il sacco di Roma, Francesco Guicciardini scrive al datario Matteo Giberti 'non ho parole pari ai concetti miei', sottolinea appunto che di fronte ad

\footnotetext{
${ }^{2}$ Come si sa, è tesi fondamentale di Machiavelli che nessuno prenda la guerra per mestiere ('usare la guerra per arte'), al di fuori di chi comanda, principe o repubblica. Per Fabrizio Colonna nell'Arte della guerra, 'essendo questa una arte mediante la quale gli uomini d'ogni tempo non possono vivere onestamente, non la può usare per arte se non una republica o uno regno; e l'uno e l'altro di questi, quando sia bene ordinato, mai non consentì ad alcuno suo cittadino o suddito usarla per arte, né mai alcuno uomo buono l'esercitò per sua particolare arte' (A I). Si vede che Machiavelli precisa subito che non c'è contraddizione nel suo pensiero tra la tesi ricordata prima e la necessità di prepararsi alla guerra e di essere capace di farla per difendersi o per acquistare, cosa che si potrebbe pensare avendo in mente il capitolo 14 del Principe. La precisazione di Fabrizio 'non la può usare per arte se non una republica o uno regno' viene ribadita: 'Debbe adunque una città bene ordinata volere che questo studio di guerra si usi ne' tempi di pace per esercizio e ne' tempi di guerra per necessità e per gloria, e al publico solo lasciarla usare per arte, come fece Roma' (A I). Ma i popoli, siano essi cittadini di 'una republica' o sudditi di 'uno regno', devono avere un mestiere che 'gli nutrisce nella pace' in modo che 'venuta la pace, [i] principi tornino a governare i loro popoli, i gentili uomini al culto delle loro possessioni, e i fanti alla loro particolare arte: e ciascuno d'essi faccia volentieri la guerra per avere pace, e non cerchi turbare la pace per avere guerra' (A I).
} 
eventi sconvolgenti mancano le parole e il processo di comprensione e di restituzione di una forma di razionalità scritta impone un'interrogazione complessa sulle parole e le frasi per dire gli esiti del conflitto armato. E la comprensione di quanto succede presuppone allora l'individuazione dei motivi per cui tutto è cominciato: la guerra va spiegata anche tramite ciò che la precede e che l'accompagna, ossia la ricostituzione dei piani, degli scopi, delle proiezioni e degli obiettivi. Parlare di guerra diventa quindi parlare di politica, perché non si tratta solo di fare la storia delle battaglie, ma di vedere se essa rimetta in forse l'esistenza della compagine statale, e perché questo discorso della guerra sia una richiesta permanente della riflessione sul vivere insieme. Machiavelli dice proprio questo nel suo battibecco con il cardinale di Roano alla fine del capitolo 3 del Principe, ma anche nel prologo dell'Arte della guerra. Nel primo caso, dire a Roano che i Francesi s'intendono forse meglio della guerra ma sicuramente meno bene dello stato significa ricordare loro che i linguaggi della guerra non sono solo quelli della zuffa armata. Nel secondo caso propone una frase che spiega meglio di qualsiasi enunciato la centralità dei capitoli 12 e 13 sulle armi nel Principe, e la richiesta fatta al principe nel capitolo 14 di pensare sempre alla guerra:

tutte l'arti che si ordinano in una civiltà per cagione del bene comune degli uomini, tutti gli ordini fatti in quella per vivere con timore delle leggi e d'Iddio, sarebbono vani, se non fussono preparate le difese loro; le quali, bene ordinate mantengono quegli, ancora che non bene ordinati. E così, per il contrario, i buoni ordini, sanza il militare aiuto, non altrimenti si disordinano che l'abitazioni d'uno superbo e regale palazzo, ancora che ornate di gemme e d'oro, quando, sanza essere coperte, non avessono cosa che dalla pioggia le difendesse. E se in qualunque altro ordine delle cittadi e de' regni si usava ogni diligenza per mantenere gli uomini fedeli, pacifici e pieni del timore d'Iddio nella milizia si raddoppiava, perché in quale uomo debbe ricercare la patria maggiore fede, che in colui che le ha a promettere di morire per lei? ${ }^{3}$

L'ordine della guerra diventa lo zoccolo dell'ordine della repubblica, le armi il puntello delle leggi. Per di più, le nuove forme della guerra, emerse con la campagna di Carlo VIII nell'autunno del 1494 nutrono e danno forma più radicale a questa logica in atto nel pensiero del Machiavelli: tali guerre sono più violente e più rapide, con effetti più dirompenti, che a

\footnotetext{
${ }^{3}$ Machiavelli 2001a, p. 27 (Proemio)
} 
loro volta condizionano i governi. Gli eserciti vi sono poi provvisti di armi nuove e s'ordinano in modo inedito sul campo. In tale prospettiva, la posta in gioco è diventata infatti la sopravvivenza stessa dello stato. I modi e le parole ricevute in eredità per pensare questi nuovi conflitti sono in gran parte obsoleti e bisogna proporre nuove modalità per dire la novità di questi tempi 'strani' (secondo le parole usate dal Guicciardini nella sua Consolatoria per qualificare la congiuntura bellica), delle guerre 'insolite' (secondo le parole del Savonarola nelle sue Prediche sopra Aggeo, per parlare della calata di Carlo VIII).

La situazione induce nei linguaggi della guerra una duplice porosità, linguistica e socio-politica. Una porosità linguistica nella misura in cui non si possono né trascurare gli effetti della varietà degli idiomi usati in eserciti che sono tutti transnazionali, né l'influenza del latino sulla lingua volgare prediletta ormai nella lingua della guerra, giacché i testi di riferimento per dire la guerra sono scritti in greco e in latino (sono i veteres scriptores de re militari, i quali sono pochi ma stampati e ristampati decine di volte in tutto il Cinquecento). Ma la porosità è anche socio-politica: per via dello sconfinamento della lingua dello scontro armato in quella della politica, le parole del racconto della guerra esterna sono suscettibili di passare nella descrizione dei conflitti interni. Il primo assunto ricorda che la lingua della guerra è sempre una lingua parzialmente ibrida; il secondo, che la lingua della guerra non è solo la lingua militare. Non bastano quindi i dizionari e i glossari per studiare questa lingua della guerra (anche se risultano ovviamente di grande utilità!). ${ }^{4}$

\section{La lingua di Machiavelli come lingua di guerra}

La lingua di Machiavelli (e chiedo scusa per questa evidenza) è una lingua della guerra prima di tutto, una lingua che impone come asse maggiore della comprensione del mondo un

\footnotetext{
${ }^{4}$ Siano essi dizionari antichi o glossari contemporanei, come quello di Michaux 2008. Si veda anche Busetto 2004.
} 
sistema guerra/pace. ${ }^{5}$ Ed è d'altronde, nei fatti, una lingua che si è costruita durante gli anni della cancelleria, quando, tra il 1498 e il 1512, Niccolò lavorava per il consiglio dei Dieci, incaricato appunto delle cose della guerra, e scrisse quindi di guerra in decine di lettere redatte quotidianamente per comunicare ordini e dare avvisi, pareri o informazioni. ${ }^{6}$ Ora, la critica ha assunto chiaramente il fatto che questo carteggio è la fucina della lingua del Principe. ${ }^{7}$ Tale lingua deve adattarsi alle caratteristiche delle guerre nuove: l'enunciato non deve perdersi in circonlocuzioni, non può ammettere la poca chiarezza, le ambiguità e le ambivalenze, deve cancellare ogni tentazione di sottigliezze e di mediazioni eccessive, per puntare direttamente al bersaglio. Frasi brevi, bilanciate, che ritagliano la realtà con distinzioni rigide e senza sfumature apparenti (si pensi al famoso procedere dilemmatico). ${ }^{8}$ Sintesi di questa convinzione è il rifiuto di una retorica asiana, illustrata da quella famosa frase della lettera dedicatoria nella quale l'autore dichiara: 'non ho ornata né ripiena di clausule ample, o di parole ampullose e magnifiche, o di qualunque altro lenocinio o ornamento estrinseco con li quali molti sogliono le loro cose descrivere et ornare'. L'unica lingua che valga deve rispettare la gravità della materia, una gravità che non è puramente astratta e non si ricollega al semplice tópos retorico classico della gravitas, ma che ha la concretezza dello scontro armato permanente. La misura di tale lingua è la sua efficacia. Si deve sempre provare a scartare le esitazioni e le approssimazioni. Si scrive e si parla per agire, e si scrive e si parla molto con modalità variegate. Machiavelli ricorre d'altronde, per narrare i tempi di guerra, alle forme di scrittura più diverse, come se fosse sempre in cerca della forma meglio adatta a dire una verità effettiva difficile da afferrare. Trattato, discorso, lettera, storiografia,

\footnotetext{
${ }^{5}$ Per una descrizione di questo sistema rimando alla nostra voce 'Pace/guerra' in Sasso 2014.

${ }^{6}$ Per un'analisi di quel modello di scrittura si veda Fournel 2006.

${ }^{7}$ Sono stati capitali in questa prospettiva i lavori di Fredi Chiappelli (1952 e 1969) non a caso fondati sulla prima edizione di una parte delle commissarie e legazioni presso l'editore Laterza. Tale studio può oggi essere ripreso ed approfondito grazie ai sette volumi di Legazioni, commissarie e scritti di governo recentemente pubblicati nell'ambito dell'edizione nazionale (Machiavelli 2002-10). Sul legame tra la scrittura di cancelleria e quella del Principe si veda Cutinelli-Rèndina 2008.

${ }^{8}$ Ma non va tuttavia esagerata la rigidità di tale procedura dilemmatica e si devono ancor meno nutrire illusioni sulla nostra capacità di rendere conto in modo esaustivo della realtà, grazie a quelle strutture binarie di pensiero.
} 
commedia, poesia didascalica, novellistica; comuni a tutte queste forme sono però, da un parte, un ritmo e un tono recisi, nonché, dall'altra, una capacità di interrogare le caratteristiche della novità e la validità dell'eredità semantica tradizionale, ossia quelle parole che sono le pietre miliari di una tradizione repubblicana niente affatto pacifica (legge, libertà, equalità, giustizia, sapienza, arti...). ${ }^{9}$

Ciò non significa minimamente, per i Fiorentini del tempo, che le parole della politica debbano cambiare, ma solo che conviene risemantizzarle e ridefinirle, o meglio ridescriverle (giacché s'impone una certa sfiducia nei confronti di definizioni astratte e chiuse) in funzione degli imperativi del momento. Non significa neppure che si debba rigettare qualsiasi enunciato troppo largo e qualsiasi parola di stampo generico - anzi, è proprio il contrario, come si vedrà dopo.

\section{Il lessico della guerra nel testo machiavelliano: distinzioni e composizioni}

Infatti nella lingua della guerra del Machiavelli (quella che va studiata non solo nell'Arte della guerra ma anche nei Discorsi, nel Principe, nelle Istorie fiorentine e negli scritti e carteggi di cancelleria) non domina sempre e soltanto il fenomeno di tecnificazione progressiva, né quello di contaminazione tra ambiti e registri diversi (secondo un'articolazione lingua comune vs. lingua specializzata). La lingua militare viene invece segnata da due fenomeni che dipendono da temporalità opposte: da un lato, la modellizzazione ancorata nel passato (diacronica) con il ruolo assunto dai latinismi dei testi di riferimento (soprattutto Vegezio e Frontino come esempi maggiori di quelli che formano i veteres scriptores de re militari, che offrono un serbatoio di parole e di esempi) ${ }^{10}$ e d'altra

\footnotetext{
${ }^{9}$ Si veda a questo proposito Fournel (2014).

${ }^{10}$ Un tale corpus è una novità nei confronti della diffusione del solo Vegezio nel Medioevo. La prima edizione collettiva che comprende i testi di Vegezio, Frontino, Eliano (tradotto dal greco in latino) e dello pseudoModesto compare nel 1487 (a cura di Giovanni Antonio Sulpizio da Veroli - Roma, Eucharius Siller - il quale ristampa il lavoro alla fine dell'anno 1494...). Un'altra importante edizione di questi testi viene curata nel 1496 da Filippo Beroaldo il Vecchio (ristampata poi nel 1505 da Giovanni Antonio de Benedictis). La prima edizione francese collettiva risale al 1515 (Parigi, Guy Breslay - ristampato a Lione presso G. Huyon nel 1523), in
} 
parte la contemporaneità radicata nel presente (sincronica), con la circolazione delle parole tra le varie lingue volgari (spagnolo, italiano, francese, tedesco, inglese) o tra le varie componenti della koiné volgare italiana. In questo modo, ad una stratificazione temporale (con parole nuove che si aggiungono a parole vecchie senza farle scomparire) fa pendant una stratificazione spaziale (con parole che dicono la stessa cosa ma vengono da territori differenziati). Tale situazione, induce a volte un sopravvento preso dalle parole che hanno una certa origine e appartengono ad un dato patrimonio lessicale quando dominano in un campo specifico i tecnici di tale o tale altra madrelingua - sarà il caso per gli ingegneri e gli architetti militari italiani nel secondo Cinquecento, così come era il caso per i canonnieri francesi nel tardo Quattrocento e nel primo Cinquecento. La lingua della guerra è infatti al contempo un lascito dell'eredità classica e il prodotto di un fenomeno storico multinazionale e, per l'Italia, multiregionale (con un posto, limitato ma presente, lasciato per esempio ad alcuni toscanismi).

Prendiamo un esempio di quell'ultima componente del linguaggio militare di Machiavelli. In un passo di D II.17, quando si parla delle 'zuffe campali', Machiavelli scrive che esse sono 'chiamate ne' nostri tempi, con vocabolo francioso, giornate, e, dagli Italiani, fatti d'arme'. Alla parola $z$ uffa di origine sconosciuta (probabilmente germanica), ma che viene considerata come fiorentina, corrispondono due parole, la parola giornata (che Machiavelli considera 'francese' poiché giornata viene secondo lui da journée - sebbene qui la logica degli andirivieni linguistici sembri mostrare che 'journée' in francese s'impone appunto solo grazie ad un 'ritorno' oltr'Alpe dell'italiano 'giornata' avvenuto appunto con le guerre d'Italia...) ed un'altra qualificata come 'italiana' con l'espressione fatto d'arme. A questo proposito, si noterà che si tratta dell'unica occorrenza di fatto d'arme nei Discorsi, una espressione che non compare né nell'Arte della guerra, né nelle Istorie fiorentine, né nel 
Principe, mentre giornata e zuffa si dividono equamente le occorrenze per nominare la battaglia (la prima parola piuttosto per le battaglie campali, la seconda piuttosto per i conflitti interni - infatti l'aggettivo campale viene raramente associato a zuffa). Machiavelli non sembra ricorrere ad una espressione ('fatto d'arme') che egli lascia agli 'italiani', mentre tale parola è invece molto frequente in Cornazzano, ma anche in Guicciardini, soprattutto nei primi libri della Storia d'Italia (dove si trova solo una volta la parola zuffa).

Nel Machiavelli la stratificazione spaziale è tuttavia nettamente meno presente di quella temporale: la dialettica più feconda nel testo machiavelliano è quella tra latino e volgare, non quella tra i diversi volgari italiani ed europei. ${ }^{11}$ In questa prospettiva, il problema maggiore della lingua militare machiavelliana è quello dell'articolazione tra l'eredità lessicale latina, la vivacità e l'innovazione lessicale contemporanea in campo militare e, infine, la porosità della frontiera tra conflitto interno e guerra esterna. Si può dire che, per il lessico della guerra nei testi machiavelliani, siamo di fronte - schematicamente - ad una triplice fonte con la tripartizione lingua generica/latinismi/lingua d'uso contemporaneo (prevalentemente tecnica). Ma si vedrà anche come, pure nella lingua tecnica e nei latinismi, esista un ricorso alla logica generica che è molto produttivo. Riprendiamo ora questi vari punti.

\section{Lingua generica: il caso-Principe}

Essa non appartiene, o non solo, al mondo della guerra guerreggiata e ci ricorda che Machiavelli (ma anche Guicciardini) non sono 'professionisti' della guerra, anzi hanno sempre considerato che la milizia moderna è solo l'ombra dell'antica e che non avevano

\footnotetext{
${ }^{11}$ I pochi studi sintetici sulla lingua della guerra nel Rinascimento, come quelli di Piero del Negro, hanno mostrato che il Cinquecento è il momento di un balzo quantitativo e qualitativo nel lessico della guerra: tutto aumenta, il numero dei neologismi, quello delle traduzioni (con un uso dell'italiano come 'lingua maieutica'), quello dei passaggi da una lingua all'altra, specialmente tra le tre lingue romanze maggiori ma anche con il tedesco e l'inglese. La poliglossia è di regola negli eserciti mercenari, tanto più quanto le giovani lingue volgari non sono ancora stabili. Inoltre, come si ricordava già sopra, le nuove guerre sono un momento di invenzioni e innovazioni tecniche e tattiche, fosse solo per il peso che vi assumono le armi da fuoco: ora la lingua della guerra non dipende mai soltanto da una trasmissione passiva e deve integrare quelle novità. In una situazione simile, $\mathrm{i}$ paradigmi dell'influenza (di una lingua sulle altre), della rivalità tra le lingue, o della gerarchia degli idiomi non bastano. Si veda Del Negro 1997 e 2002.
} 
granché da imparare dai capitani del presente. Nella lingua per la guerra dei fiorentini del tempo, non solo nel Machiavelli, si nota infatti un uso massiccio di un lessico generico della guerra che non è ancorato in un lessico tecnico. Tale uso viene illustrato da parole come amore, armi (ovviamente), cose di dentro e cose di fuora, disciplina, esercizio (il più delle volte adoperato in un contesto militare - P 10, 14, 21), esercito, fianco, genti, giustizia, milizia, ${ }^{12}$ necessità, odio, ordine, terra e tante altre parole.

Lo spazio specifico di quella lingua generica (la quale è presente in tutti i testi), è giust'appunto Il Principe. Infatti nell'opuscolo le armi sono sempre presenti e sono al cuore del ragionamento, ma tale ragionamento si cristalizza attorno a quattro questioni maggiori, nessuna delle quali viene trattata con un lessico prevalentemente tecnico: i mercenari come questione politica-economica-morale; la capacità militare del principe nuovo; l'articolazione tra leggi e armi; infine, il sopravvento della politica estera su quella interna con l'emergenza del terzo umore dei soldati. ${ }^{13}$

Il Principe dice quindi, in fin dei conti, poco attorno alla questione militare come componente strettamente tecnica delle pratiche militari. Si pensano invece nel Principe la disciplina dell'esercito, l'inimiciza strutturante delle relazioni politico-militari (bisogna sapere essere vero amico o vero inimico, secondo quanto viene enunciato nel capitolo 20), ${ }^{14}$ la necessità di una forza minima (nel capitolo 10), la forza della religione (nel capitolo 11) ma anche la forza che toglie la religione (capitolo 12), la composizione delle truppe, la neutralità o il sistema delle alleanze, il sapere, la virtù e la fortuna dei capitani e dei principi, la

\footnotetext{
${ }^{12}$ La parola milizia viene usata abbastanza spesso, ossia una quarantina di volte nell'Arte della guerra e una ventina nei Discorsi, ma due volte soltanto nelle Istorie fiorentine. La parola militi è usata solo una volta nei Discorsi e un'altra nell'Arte della guerra: Machiavelli sceglie accuratamente i suoi latinismi.

${ }^{13}$ Non è un caso se quell'introduzione dei soldati come terzo umore compare alquanto tardi nel testo, nel capitolo 19, come una delle maggiori tracce dell'autogenerazione del testo del Principe, ossia della capacità dell'autore di fare nascere dal testo stesso alcuni momenti dell'argomentazione senza che essi siano stati preventivati e pensati nello schema iniziale del lavoro.

${ }^{14}$ La cosa era stata chiaramente messa a fuoco per Machiavelli durante le sue legazioni, come dimostra l'uso della coppia amico/nemico nelle sue lettere mandate dalla corte di Cesare Borgia o da quella di Luigi XII. Così, il 18 luglio del 1510, in un sua lettera ai Dieci, Machiavelli racconta come Luigi XII gli dichiarò: 'io voglio sapere chi è mio amico o mio inimico’' (Machiavelli 1999, p. 1257).
} 
conoscenza dei siti, l'amore dei soldati per il loro capo, la mancanza di virtù militare in questi nostri corrotti tempi, la giustizia e il tempo delle armi.

Ma non ci si sofferma molto - tra altre questioni maggiori - sul reclutamento (il famoso 'deletto' dell'Arte della guerra e il nodo cruciale dell'ordinanza nel 1506), sull'artiglieria (non allude una sola volta ad essa nel testo), o più generalmente sulle armi da fuoco (quale il famoso scoppietto di cui tesse l'elogio nell'Arte della guerra), sulla nominazione delle armi, sugli ordini dell'esercito prima della battaglia, sulle fortificazioni campali, e neanche sull'organizzazione pratica dell'economia del mercenariato ecc.

È vero che ci sono anche poche ma decisive eccezioni, ma ogni volta esse vengono ridimensionate non appena sono state espresse. Prendiamone alcuni esempi. Il riferimento rapido alla creazione di una milizia di fanti e arcieri da parte del re di Francia Carlo VII nel capitolo 13, serve solo a condannare l'abbandono, da parte della Francia, della strada giusta di un esercito nazionale. Il trattamento della questione delle fortezze nel capitolo 20 risulta un ennesimo modo per ribadire la necessità di avere il popolo amico e, in fin dei conti, il tema della fortezza risulta qui alquanto strumentale. L'opposizione fanteria/cavalleria, che compare dapprima nella genealogia schematica delle compagnie di ventura italiane nel capitolo 13, è il caso forse più interessante, perché viene ripresa solo nelle ultime pagine del libro, nell'Exhortatio dell'ultimo capitolo con il ragionamento sul terzo ordine della fanteria in grado di resistere sia alla cavalleria che ad un'altra fanteria; ciononostante, Machiavelli non intende entrare qui nel discorso sulla 'generazione delle arme e la variazione delli ordini' (P 26), ossia sulla natura degli armamenti (picca, spada, scoppietto ecc.) e sull'ordinamento delle file di combattenti sul campo (quanto verrà descritto nel terzo libro dell' Arte della guerra per filo e per segno). Si può presumere che l'autore voglia infatti rimandare ad un'altra opera 'quelle cose che, di nuovo ordinate, danno reputazione e grandezza a uno principe nuovo' (P 26). 


\section{Il tempo delle parole: il confronto con il latino}

Ciononostante, l'articolazione del sapere tratto dall'esperienza diretta della guerra con la convinzione che la milizia moderna sia inferiore a quella antica spinge Machiavelli (soprattutto nell'Arte della guerra - e quasi mai nel Principe) a lasciare uno spazio peculiare al lessico della guerra di origine latina e a pensare il posto di esso nei confronti di un lessico più contemporaneo (sia esso generico o tecnico e specializzato). Il complesso sistema dei volgari moderni, che nello stesso momento storico s'instaura appunto in articolazione con il latino, conferisce d'altronde ai latinismi un posto specifico, specialmente nella redazione della storia o dei dialoghi o trattati didattico-morali.

Machiavelli sviluppa un suo uso peculiare del latinismo, come si vedrà con l'esempio di deletto o di asta). I latinismi vengono messi quindi a confronto con una lingua tecnica sempre più copiosa, creata per descrivere le realtà inedite degli armamenti e delle campagne o pratiche militari, con forme di mediazioni e di negoziati spontanei per alcune parole della lingua d'uso, con traduzioni-adattamenti immediati di termini che diventano neologismi nell'altra lingua, nella lingua d'arrivo, ma con sensibile colore straniero: Machiavelli è profondamente consapevole di quel fattore di ulteriore complessità sorto con il passaggio generalizzato dal latino al volgare. Risponde ai problemi posti adoperando, a seconda dei testi

e dei contesti argomentativi, varie soluzioni compatibili e complementari. Lo studio della lingua della guerra e della lingua militare in Machiavelli non può essere fatto solo alla luce di una delle sue opere maggiori: è importante tentare di dire quali siano le differenze a questo proposito tra le opere (ivi compresi quei testi che non sono vere e proprie opere, come le legazioni o commissarie). Non ci si può neanche accontentare di dati quantitativi (per esempio l'abbondanza numerica dei latinismi lessicali nell'Arte della guerra e il loro numero limitato negli altri testi): bisogna infatti prendere in considerazione un uso qualitativo dei latinismi. 
Il posto dei latinismi militari nell'Arte della guerra può essere illustrato dall'esempio del termine deletto, tratto dal latino militare, il quale rimanda al reclutamento dei fanti per costituire una milizia (proposta, si sa, molto cara al Machiavelli dall'ideazione dell'ordinanza fiorentina del 1506, e proposta che nutre anche la riflessione tattica militare dei capitoli 12-13 e 26 del Principe). Ora, deletto nell'Arte della guerra non compare né come il solito latinismo (come modifica limitata di una parola latina trasferita in volgare ma destinata ad uso comune), né come un calco (come trascrizione passiva), ma si configura come una ripresa dinamica e singolare di una parola che ha un peso tale da non potere essere trasmessa da un semplice atto di traduzione (viene rimandata esplicitamente ai Romani con l'espressione 'il deletto di essi, che così lo chiamavano gli antichi'). La parola, per cristallizzare una forza ed un significato maggiori, appare addirittura come 'bruciata' non appena viene usata: dopo una trentina di occorrenze, quasi tutte nel primo libro dell'Arte della guerra, quando si tratta la questione politica delle armi proprie, vi si ricorre solo un'altra volta nel dialogo, e due volte nei Discorsi (D III.30 e 33). In tutte le altre opere di Machiavelli e nelle opere militari del tempo che ho potuto consultare l'uso di deletto non compare.

La stessa analisi potrebbe essere fatta attorno ad un termine ancora più tecnico, l'asta (che appare anch'esso solo nell'Arte della guerra), e alle altre parole che rinviano a questo tipo di armi. Machiavelli scrive a questo proposito in tre momenti diversi del dialogo: prima ricorda che i Romani 'avevano uno dardo in mano, il quale chiamavono pilo, e nello appiccare la zuffa lo lanciavano al nimico' (A II); poi in un altro passo (A II) dichiara che 'le falangi di Macedonia, portavano aste che chiamavono sarisse, lunghe bene dieci braccia, con le quali eglino aprivono le stiere nimiche e tenevano gli ordini nelle loro falangi'; in fine, ancora più in là, quanto al 'modo dello armare presente', afferma (A II) che 'hanno i fanti, per loro difesa, uno petto di ferro e, per offesa, una lancia nove braccia lunga, la quale chiamano picca, con una spada al fianco piuttosto tonda nella punta che acuta'. Si nota quindi ogni volta 
un'esitazione tra due termini (uno generico e uno tecnico e storicamente determinato; dardo/pilo; asta/sarissa; lancia/picca) con la chiara consapevolezza che esistono ad ogni epoca problemi di nominazione (come dimostra la ricorrenza del verbo chiamare). ${ }^{15}$

L'Arte della guerra è dunque il luogo specifico di uso dei latinismi vivaci, ma il Machiavelli è consapevole di farne un uso che gli è proprio, lasciando uno spazio linguistico al lessico latino tecnico (come se volesse ridare vita a queste parole latine), ma anche limitando i latinismi ad alcuni casi e momenti specifici. In questo modo i latinismi sono illustrazioni della temporalità mutevole delle parole, del fatto che esse hanno una vita loro, o invece del fatto che esse possono rinchiudersi in una morta testualità puramente libresca. Non basta riprendere le parole se non si capisce la natura del collegamento tra queste e la storia del tempo presente; le parole, quando non corrispondono più a nessuna realtà, possono morire o diventare semplici tracce dei morti del passato lontano, suscettibili di interessare solo quegli antiquari criticati nel proemio dei Discorsi. Così nella lingua militare di Machiavelli non esistono sempre parole corrispondenti per tutte le parole degli antichi e, simmetricamente, egli non ammette le false corrispondenze dei moderni. ${ }^{16}$

La dimostrazione più chiara delle specificità della lingua militare machiavelliana nell'Arte della guerra e nei Discorsi o nel Principe sta, a contrario, nell'esame che si può fare del lessico militare delle lettere di cancelleria. In quelle lettere Machiavelli non esita ad adoperare un lessico più tecnico. Nelle lettere di cancelleria è anche molto presente ovviamente il lessico della condotta (il quale stranamente non compare nelle più acerrime denunce dei mercenari nel Principe): di condotta o condottiere si trovava una sola occorrenza nel Principe e solo una quindicina nelle Istorie fiorentine, mentre nelle lettere di cancelleria se ne trovano più di 300. Simili considerazioni potrebbero essere fatte a proposito di termini

\footnotetext{
${ }^{15}$ Nel libro III Machiavelli riprenderà di passaggio questo esempio, scrivendo: 'le loro lance, le quali chiamavano sarisse erano sì lunghe che la sesta fila passava con la punta della sua lancia fuora della prima fila' (A III), ciò che mostra una vera esitazione per il termine generico tra lancia e asta.

${ }^{16}$ Per ragioni di spazio rimando, per un ulteriore sviluppo di questo tema, ad un altro lavoro in corso.
} 
come lancia, rivellino, tagliata, ripari, bastione. Invece, come ci si poteva aspettare, Machiavelli non usa mai nelle lettere di cancelleria il lessico tecnico direttamente preso in prestito dal latino, che sarà invece molto frequente nell'Arte della guerra (penso per esempio a parole come dardo, pilo e sarissa; nel carteggio appare solo una volta asta, mai caterva e legione). Ma l'esempio più lampante della capacità di Machiavelli di tirare in ballo il lessico tecnico del proprio tempo, sta nel parlare che fa di artiglieria o di armi da fuoco: nel carteggio di cancelleria distingue tra archibuso, bombarde grosse, bombardelle, cannone, cortaldo, falconetto, passavolante, spingarda.$^{17}$ In quelle lettere si può però anche dare al termine di artiglieria un significato generico (ancora più generico di quello che gli è conferito nell'Arte della guerra, dove il termine viene usato per ogni tipo di cannone, e anche per ogni arma da fuoco con l'eufemismo di artiglieria minuta, a sostituire la sfilza dei nomi tecnici dei cannoni e armi da fuoco): ${ }^{18}$ questo significato ultragenerico (dove artiglieria designa tutto ciò che è necessario per l'uso delle armi da fuoco) si legge nella frase: 'Le artiglierie che ieri e stamani abbiamo inviate a cotesta volta son queste: 10 archibusi, 3 barili di polvere, 391 libbra di piombo, 5 casse di passatoi, 3 code di spingarde, e inoltre una soma di piombo'. ${ }^{19}$

Simmetricamente, il trattamento del termine artiglieria nell'Arte della guerra, così come è stato ricordato prima, e dei termini collegati con il lessico delle armi da getto e da fuoco, propone, con la scelta di usare un'unica parola per tutti i tipi di cannoni e quasi tutti i tipi di armi da fuoco, un esempio interessante del modo di sviluppare la logica generica. A questa onnipresenza del termine artiglieria fa eccezione - e pendant - nell'Arte della guerra la parola scoppietto, secondo una modalità che aiuta a lanciare ponti tra logica tecnica e logica

\footnotetext{
${ }^{17}$ Niccolò Machiavelli al capitano e commissario di Livorno, 12 luglio 1503, in Machiavelli 2002-10, vol. 3, p. 187. Vedi anche la voce 'Artiglieria' che abbiamo redatto per Sasso 2014.

${ }^{18}$ Una volta sola cannone viene usato nell'Arte della guerra (A III) in senso più tecnico, con precisazioni sul calibro, la portata ed il peso: 'L'artiglierie dell'esercito, bastano dieci cannoni per la espugnazione delle terre, che non passassero cinquanta libbre di portata; de' quali in campagna mi servirei più per la difesa degli alloggiamenti che per fare giornata; l'altra artiglieria tutta fusse piuttosto di dieci che di quindici libbre di portata. Questa porrei innanzi alla fronte di tutto l'esercito, se già il paese non stesse in modo che io la potessi collocare per fianco in luogo securo, dov'ella non potesse dal nimico essere urtata'.

${ }^{19}$ Machiavelli ai Commissari di Poppi, 6-17 novembre 1498 (Machiavelli 2002-10, vol. 1, pp. 125-6).
} 
generica al di là della questione dei tempi (e del confronto tra lingua antica e moderna). L'uso di scoppietto potrebbe sembrare contradditorio con il tropismo generico di cui si parlava sopra. Ma non lo è, giacché, per i Fiorentini, la parola viene spesso adoperata per additare tutte le armi da fuoco portatili. Questo spiega anche l'assenza nell'Arte della guerra di un termine come archibuglio o archibuso: Machiavelli non ne ha bisogno, non rifiuta di usare tali termini ma preferisce a loro una parola più naturale che è immediatamente disponibile, quella di scoppietto giust'appunto. E, a ulteriore dimostrazione di ciò, nella sua traduzione francese dell'Arte della guerra Jean Charrier 'ristabilisce' la parola arquebuse per tradurre scoppietto. ${ }^{20}$ Inoltre quando usa le parole scoppietto e scoppiettieri (una diecina di volte nell'Arte della guerra), Machiavelli insiste sull'importanza di quell'arma moderna chiamandola 'istrumento nuovo - come voi sapete [è quindi una dóxa ammessa da tutti] - e necessario' (A II). Si potrebbe tornare in modo proficuo, a questo proposito, sulle troppo frettolose condanne di Machiavelli da parte degli specialisti della cosa militare, come uno che non capì niente delle armi moderne: Fabrizio Colonna vuole infatti nelle file del suo esercito un numero cospicuo di scoppiettieri (1000 ogni 6000), i quali sul campo sono più utili delle artiglierie pesanti. ${ }^{21}$ L'inserzione delle armi da fuoco nella fanteria trasforma in questo modo lo scoppietto - come la picca - in un'arma dei popoli poveri e liberi, che combattono i nobili a cavallo. $^{22}$

Lo sfoggio di conoscenza delle macchine da getto e del lessico della poliorcetica in uso presso i Romani va letto, a contrario, come un'ulteriore illustrazione della stessa articolazione sofisticata tra lingua generica e lingua tecnica. Machiavelli scrive nell'Arte della

\footnotetext{
${ }^{20}$ Machiavelli 1546, p. $18 \mathrm{v}$ e p. $24 \mathrm{r}$.

${ }^{21}$ A III, p.134: 'vero è che assai più nuocono gli scoppietti e l'artiglierie minute, che quelle'. Vedi anche Arte della guerra I, pp. 68-9: 'dico che Pompeo e Cesare, e quasi tutti quegli capitani che furono a Roma dopo l'ultima guerra cartaginese, acquistarono fama come valenti uomini, non come buoni; e quegli che erano vivuti avanti a loro, acquistarono gloria come valenti e buoni. Il che nacque perché questi non presero lo esercizio della guerra per loro arte, e quegli che io nominai prima, come loro arte la usarono'; si veda anche D III.25 e A I: 'I miei Romani, come ho detto, mentre che furono savi e buoni, mai non permessero che i loro cittadini pigliassono questo esercizio per loro arte'.

${ }^{22}$ Si noterà en passant che l'inserzione delle armi da fuoco nelle file di fanti viene qui attribuita ai Tedeschi e agli Svizzeri più che agli Spagnoli, contrariamente a quanto viene per lo più sostenuto nella storiografia militare.
} 
guerra, VII: 'Gli instrumenti co' quali gli antichi difendevano le terre erano molti, come baliste, onagri, scorpioni, arcubaliste, fustibali, funde; ed ancora erano molti quegli co' quali le assaltavano, come arieti, torri, musculi, plutei, vinee, falci, testudini. In cambio delle quali cose sono oggi l'artiglierie, le quali servono a chi offende e a chi si difende; e però io non ne parlerò altrimenti', elenco che riprende pari pari quello di Vegezio (A IV), e che mostra che Machiavelli cita queste macchine più per mostrare di conoscerle che per adattarle al mondo moderno. Questo lessico antico è morto, interessante solo per quegli antiquari derisi nel prologo dei Discorsi. Una sola parola basta per queste tredici parole: artiglieria. D'altronde Machiavelli non intende tornarre su tale elenco ('e però io non ne parlerò altrimenti') e neanche esplicitare i significati di parole non chiarissime per uno che non abbia letto Vegezio - solo la parola torri, per motivi ovvi, si ritrova adoperata nelle altre opere del Segretario fiorentino e neanche la parola ariete, di uso comune, lo è.

Da quell'esempio possiamo inferire che il lessico generico non è portatore d'approssimazioni, ma può condurre a precisare parole, usi e tattiche. Se le cose vanno così, lo si può ricondurre alla convinzione semplice ricordata sopra, secondo la quale se il militare e il civile sono indissociabili (come ricordano i capitoli 12-14 del Principe e il prologo dell'Arte della guerra), non significa che vadano confusi, giacché l'esercizio della guerra non deve essere per i cittadini o i sudditi (non è così per i capitani e i principi) un'arte, un mestiere (è quel che ricorda Fabrizio fin dall'inizio del dialogo). Insomma Machiavelli, in questa prospettiva, non trascura la precisione delle parole quando occorre, ma non ne fa una condizione dell'enunciato sempre e comunque. L'unica cosa che conta è l'effetto dell'enunciato nella situazione specifica ed esso presuppone a volte una lingua non propria del solo campo militare, una lingua apparentemente più generica o che sa giocare con la genericità degli elementi di lessico adoperato (siano essi tecnici, latineggianti o di altro tipo), ma che è molto semplicemente la lingua dello stato come spazio di dispiegamento della 
politica come conflitto. In questo modo le soluzioni proposte da Machiavelli sono diverse a seconda dei momenti e delle opere, con forme di equilibrio sofisticato tra i vari lessici. L'unico criterio di quell'arte della lingua che si mette al servizio dell'arte dello stato è l'efficacia. La situazione d'emergenza e di guerra permanente non induce il sopravvento delle cose sulle parole (contrariamente a quanto viene a volte frettolosamente opinato), ma richiede un nuovo tipo di collegamento tra esse. La scelta fondamentale delle armi proprie non significa solo ricorrere a truppe non mercenarie, ma anche ad armi che siano proprie dell'ordine repubblicano, di un certo tipo di comunità politica; e a quelle armi proprie corrispondono parole proprie. 


\section{Bibliography}

Allmand, Christopher 2011, The De Re Militari of Vegetius: The Reception, Transmission and Legacy of a Roman Text in the Middle Ages, Cambridge: Cambridge University Press.

Bayley, Charles Calvert 1961, War and Society in Renaissance Florence: the De militia by Leonardo Bruni, Toronto: University of Toronto Press.

Busetto, Riccardo 2004, Il dizionario militare: Dizionario enciclopedico del lessico militare, Bologna: Zanichelli.

Chiappelli, Fredi 1952, Studi sul linguaggio di Machiavelli, Firenze: Le Monnier.

— 1969, Nuovi studi sul linguaggio di Machiavelli, Firenze: Le Monnier.

Cutinelli-Rèndina, Emanuele 2008, 'Gli scritti di governo nella genesi del Principe', in Governare a Firenze, a cura di Jean-Louis Fournel e Paolo Grossi, Paris: Belin.

Del Negro, Piero 1997, 'Tra Italia ed Europa: La guerra nello specchio della lingua', in $I$ Farnese: Corti, guerra, e nobiltà in antico regime, a cura di Antonella Bilotto et alii, Roma: Bulzoni.

— 2002, 'Una lingua per la guerra: il rinascimento militare italiano', in Storia d'Italia. Annali 18. Guerra e Pace, Torino: Einaudi.

Fontaine, Marie-Madeleine e Jean-Louis Fournel 2014, Langue militaire et langues de la guerre, Eurolab/Laboratoire des langues vulgaires dans l'Europe de la Renaissance, Genève: Droz.

Fournel, Jean-Louis 2006, 'Temps de l'écriture et Temps de l'Histoire dans les écrits de gouvernement de Machiavel', in Machiavelli senza i Medici : Scrittura del potere/Potere della scrittura, a cura di Jean-Jacques Marchand, Roma: Salerno.

— 2009, 'Florence (1494-1530): une république de guerre', in La République dans tous ses états, sous la direction de Claudia Moatti et Michèle Riot-Sarcey, Paris: Payot.

— 2014, 'L'instabile stabilità della lingua della politica. Note sulla durata semantica delle parole', in Miscellanea di studi in onore di Amedeo Quondam, a cura di Beatrice Alfonzetti et alii, Roma: Bulzoni (in corso di stampa).

Fournel, Jean-Louis and Jean-Claude Zancarini 2008, La Grammaire de la république: Langages de la politique chez Francesco Guicciardini, Genève, Droz.

Guicciardini, Francesco 1988, Avertissements politiques/Ricordi, sous la direction de JeanLouis Fournel et Jean-Claude Zancarini, Paris: Le Cerf.

- 1996, Histoire d'Italie (Storia d'Italia), sous la direction de Jean-Louis Fournel et JeanClaude Zancarini, in two volumes, Paris: Laffont. 
— 1997, Ecrits politiques, sous la direction de Jean-Louis Fournel et Jean-Claude Zancarini, Paris: PUF.

Hörnkvist, Mikaël 2010, 'Machiavelli's military project and the Art of War', in The Cambridge Companion to Machiavelli, edited by John Najemy, Cambridge: Cambridge University Press.

Machiavelli, Niccolò 1546, L'Art de la guerre, composé par Nicolas Machiavelli, ... L'Estat aussi et charge d'un lieutenant général d'armée, par Onosander, ... Le tout traduict en vulgaire françois, par Jehan Charrier, Paris: J. Barbé.

— 1999, Opere, vol. II, a cura di Corrado Vivanti, Turin: Einaudi.

— 2000, De principatibus - Le Prince, traduction et commentaire de Jean-Louis Fournel et Jean-Claude Zancarini; texte italien établi par Giorgio Inglese, Paris: PUF.

- 2001a, Arte della guerra, in L'Arte della guerra: Scritti politici minori, a cura di JeanJacques Marchand, Denis Fachard e Giorgio Masi, Rome: Salerno.

- 2001b, Discorsi sopra la prima deca di Tito Livio, in due volumi, a cura di Francesco Bausi, Roma: Salerno.

- 2002-10, Legazioni, commissarie e scritti di governo, in sette volumi, a cura di JeanJacques Marchand et alii, Roma: Salerno.

— 2014, De principatibus - Le Prince, traduction et commentaire de Jean-Louis Fournel et Jean-Claude Zancarini; texte italien établi par Giorgio Inglese, Paris: PUF.

Michaux, Marie-Anne 2008, Glossaire des termes militaires du seizième siècle: Complément du Dictionnaire d'Edmond Huguet, Paris: Champion.

Parrott, David 2012, The Business of war: Military enterprise and Military Revolution in early modern Europe, Cambridge: Cambridge University Press.

Richardot, Philippe 1998, Végèce et la culture militaire au Moyen Age, Paris: Economica.

Sasso, Gennaro (a cura di) 2014, Enciclopedia machiavelliana, Roma: Treccani. 
